\title{
An improved method for predicting iron losses in SMC electrical machines
}

\author{
Y.G. GUO ${ }^{\mathrm{a}, *}$, J.G. ZHU ${ }^{\mathrm{a}}$, J.J. ZHONG ${ }^{\mathrm{a}}$, P.A. WATTERSON ${ }^{\mathrm{a}}$ and W. WU ${ }^{\mathrm{b}}$ \\ ${ }^{a}$ Faculty of Engineering, University of Technology, Sydney, P.O. Box 123, Broadway, NSW 2007, Australia \\ * Tel.: +61 29514 7903; Fax: +61 29514 2435; E-mail: youguang@eng.uts.edu.au \\ ${ }^{b}$ CSIRO Telecommunications and Industrial Physics, P.O. Box 218, Lindfield, NSW 2070, Australia
}

\begin{abstract}
This paper presents an improved method for predicting iron losses in electrical machines with soft magnetic composite (SMC) cores. Different formulations are used for iron loss prediction with purely alternating, purely circular rotating, and elliptically rotating flux density vectors, respectively. A series of three-dimensional (3D) finite element analyses is conducted to determine the flux density locus in each element in a claw pole permanent magnet SMC electrical machine when the rotor rotates. The predicted results are compared with the experimental data of the prototype.
\end{abstract}

Keywords: iron loss, SMC, electrical machine, finite element analysis.

\section{Introduction}

The local flux density patterns within a rotating electrical machine are quite complex. The flux density locus at one position can be alternating (1D) with or without harmonics, two dimensional (2D) or even 3D rotating with purely circular or elliptical patterns. Experiments on samples revealed significant difference between the core losses caused by alternating and by rotating magnetic fields.

This paper outlines the core loss calculation in a claw pole SMC machine by using a 3D finite element analysis of magnetic field. The total core loss is computed by summing up the core loss of each element, which can be obtained by calculating separately the hysteresis (alternating and rotational, both purely circular and elliptical), eddy current, and anomalous losses, when the rotor rotates. The coefficients for each loss component are determined by a loss separation procedure and the experimental data obtained by using a single-sheet two-dimensional core loss testing system [1].

\section{Modelling of iron losses}

For alternating core loss modelling, a standard practice is to separate the loss into three components: hysteresis, eddy current and anomalous losses, i.e. $P_{a}=C_{h a} f B^{h}+C_{e a}(f B)^{2}+C_{a a}(f B)^{1.5}$, where $B$ is the peak value of flux density, $f$ the frequency, and $C_{h a}, h, C_{e a}$, and $C_{a a}$ are the loss coefficients.

Similarly, the specific core loss with a circular rotating flux vector $\boldsymbol{B}$ can also be separated into three components as $P_{r}=P_{h r}+C_{e r}(f B)^{2}+C_{a r}(f B)^{1.5}$, where $P_{h r}$ is the rotational hysteresis loss, and $C_{e r}$ and $C_{a r}$ are coefficients for the rotational eddy current and anomalous loss components. The rotational hysteresis loss behaves very differently from its alternating counterpart. A rotational field causes twice the loss due to an alternating field with the same peak value at a mid-range of flux densities but at the saturation level the loss caused by a rotating field falls markedly to the levels well below that caused by an alternating field. To 
model the rotational hysteresis loss, a formulation was proposed in [2]. It is postulated that the specific rotational hysteresis loss per cycle can be expressed in terms of four parameters, $a_{1}, a_{2}, a_{3}$, and $B_{s}$, by

$$
\frac{P_{h r}}{f}=a_{1}\left[\frac{1 / s}{\left(a_{2}+1 / s\right)^{2}+a_{3}^{2}}-\frac{1 /(2-s)}{\left[a_{2}+1 /(2-s)\right]^{2}+a_{3}^{2}}\right] \text {, where } s=1-\frac{B}{B_{s}} \sqrt{1-\frac{1}{a_{2}^{2}+a_{3}^{2}}}
$$

More generally, the flux density loci in 3D flux machines may be elliptical, characterised by major and minor axes $B_{m a j}$ and $B_{\min }$, with ratio $R_{B}=B_{m i n} / B_{m a j}$, with alternating $\left(R_{B}=0\right)$ and circular $\left(R_{B}=1\right)$ patterns as limiting cases. The core loss with an elliptical rotating flux density can be predicted from the alternating and purely circular formulations as [2]: $P_{t}=R_{B} P_{r}+\left(1-R_{B}\right)^{2} P_{a}$, where $P_{r}$ and $P_{a}$ are the core losses with a circular $\boldsymbol{B}$ and an alternating $\boldsymbol{B}$ with $B_{m a j}=B_{p}$, respectively, and $B_{p}$ the peak value of the alternating $\boldsymbol{B}$.

\section{Magnetic field analysis and core loss calculation}

Figure 1 illustrates the magnetically relevant parts of the rotor and the stator of a three-phase claw pole SMC motor. The rotor consists of a mild steel case, three arrays of NdFeB magnets, and two aluminium end plates. The stator has three stacks and each consists of two pieces of SMC claw pole discs. The stator teeth (claw poles) of three stacks are shifted by two-thirds of a tooth-pitch or 120 electrical degrees. In contrast, the magnets with the same polarity of three arrays line up respectively.

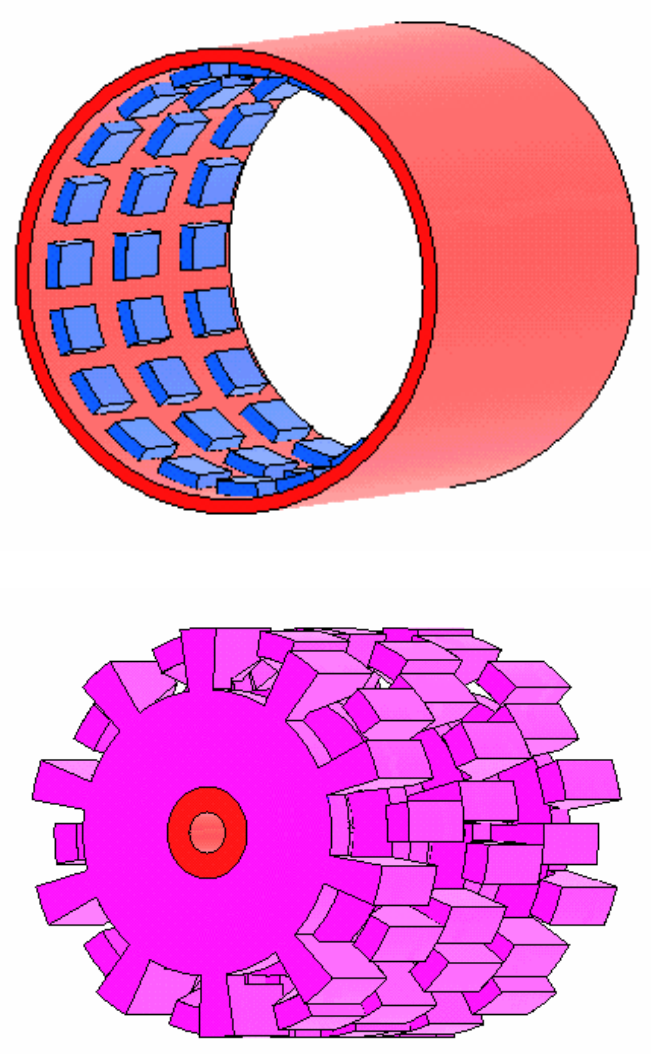

Fig. 1. Magnetically relevant parts of claw pole motor

Because of the complex geometry, the magnetic field in the armature takes all three directions. Lamination is difficult and soft magnetic composite material becomes preferable. For the correct calculation of magnetic field and motor characteristics, a 3D numerical analysis is required. Figure 2 shows 
the calculated time variations of the flux density at a typical element a claw pole. Further analysis shows that the locus of $\boldsymbol{B}$ rotates elliptically in a plane, which is not parallel to any coordinate axis. Figure 3 shows the core loss calculation summed over all elements for different frequencies or rotor speeds.

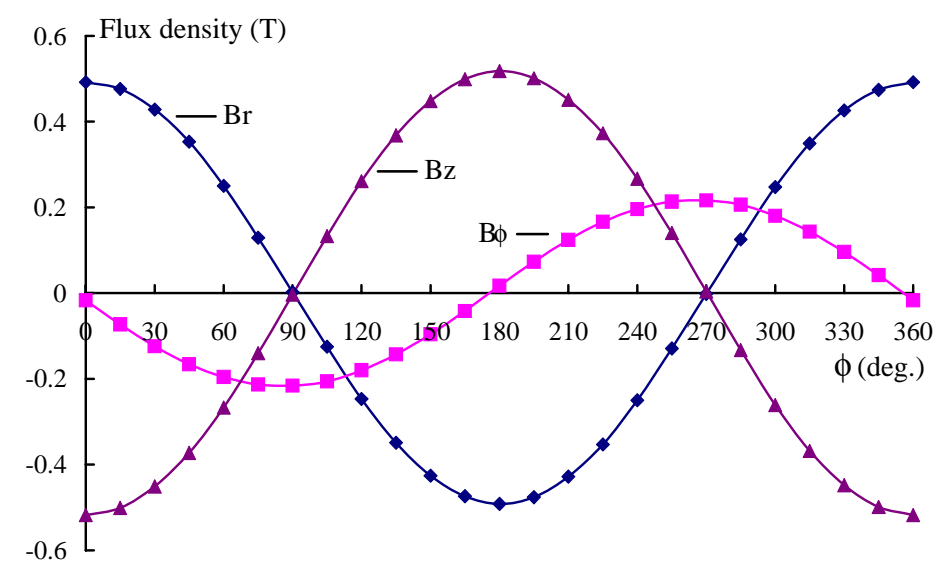

Fig. 2. Time variation of flux density at a typical point in the claw poles

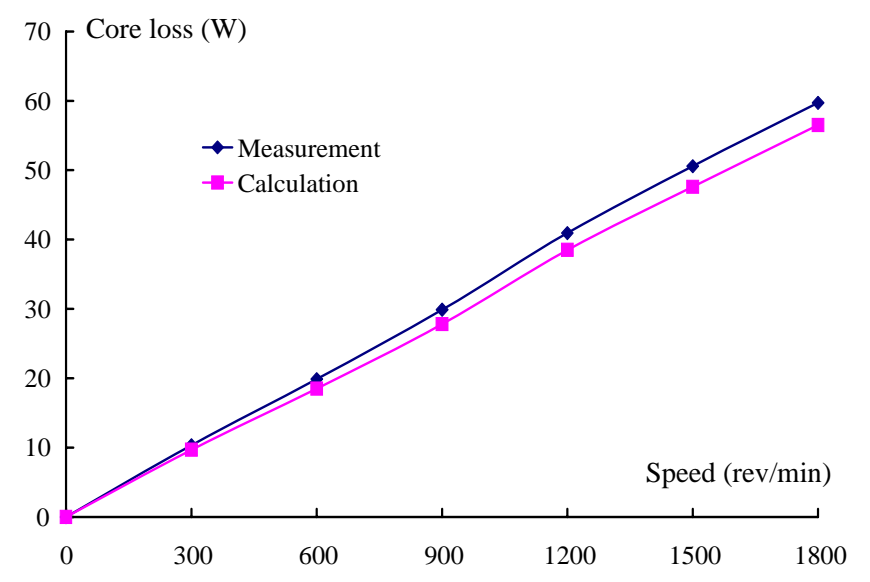

Fig. 3. Core loss calculation and measurement

\section{Core loss measurement}

To measure the core loss of the claw pole PM machine, a calibrated dc motor is used as a prime mover. At no-load and connected to the tested machine, the power fed into the dc motor is measured. The difference gives the sum of the core loss and mechanical loss of the tested machine.

The mechanical loss of the PM machine is measured by replacing the SMC stator with a wood tube (to imitate the windage) and then repeating the previous procedure. The core loss is obtained by subtracting the mechanical loss from the sum of the core loss and mechanical loss. The measured core losses at different speeds are also plotted in Figure 3. 


\section{Conclusions}

The comparison between the calculated and measured core loss in a claw pole permanent magnet SMC machine shows that the proposed core loss models and calculation method are practical. The calculation core loss is about $6 \%$ lower than the measured. This may be due to iron loss in the stator shaft and rotor tube.

\section{Acknowledgement}

The authors wish to thank Höganäs AB, Sweden for providing the preforms of SOMALOY'M 500.

\section{References}

[1] J.G. Zhu, J.J. Zhong, V.S. Ramsden, and Y.G. Guo, Power losses of composite soft magnetic materials under two dimensional excitations, Journal of Applied Physics, USA, Vol.85, No.8, April 1999, pp.4403-4405.

[2] J.G. Zhu and V.S. Ramsden, Improved formulations for rotational core losses in rotating electrical machines, IEEE Transactions on Magnetics, Vol.34, No.4, July 1998, pp. 2234-2242. 